\title{
The Impact of Feasibility Study in Enhancing Growth and Development of Business Organisations in Nigeria
}

\author{
Mohammed Alhaji Audu \\ Department Of Finance University Of Maiduguri, Borno State, Nigeria
}

\begin{abstract}
The need as to the relevance of feasibility study in the structure of a business has attracted attention from both the business circle as well as academics. It has often been concluded that a business can thrive without undergoing a feasibility study. A healthier debate is that there are no available statistics of many businesses that went under unnecessarily due to lack of such a study, thereby negating economic growth. This paper examines the impact of feasibility study in enhancing growth and development of business organizations in Nigeria. It was proven that a well planned feasibility studies enables the business owner to understand the schematics of the venture and boost confidence in facing challenges that may arise in the business life circle because the target, through feasibility study has been attained; howbeit abstract. A man who knows where he is going arrives there no matter the obstacles. The work concluded that feasibility study impact significantly on the growth of a business and reduces the level of exposure to risk and ensures success.
\end{abstract}

Keywords: Feasibility, Growth, Planned, Risk, Venture

\section{Introduction}

Feasibility study is an investigation carried out to access the economic viability of any proposed business. Every business establishment has a possibility of success or failure. Therefore, it is the responsibility of a prospective entrepreneur to know very well about the business. An intended business success or failure could be determined by the amount of information that he has before engaging in a particular venture. This therefore brings to the mind the issue of feasibility study. Feasibility study is an assessment of a project or business opportunity to determine whether or not it is worthy of undertaking. It helps the investor to sharpen his judgment and not replace it. All businessmen are advised to know all that they are capable of knowing about the enterprise, the product or service that they want to provide and the only way of doing this is by carrying out a well prepared feasibility studies. This should be done by the businessman.

Carrying out any technical study involves planning. Planning reduces the degree of risk in operation or organization by providing information concerning the circumstances and facts that influence success or failure of a proposed course of action. Planning is deciding in advance what to do, when to do it and who to do it. It is also a set of managerial activities designed to prepare the enterprise for the future and ensure that decisions regarding the use of people and resources help achieve the objectives of the enterprise; planning helps to reduce uncertainty within an organization.

The need for feasibility study is that for projects to be feasible yesterday at a given location does not mean that they would be so today for the same or different location given the erratic changes that occur now and then in the economy and that all projects are unique in their own ways.

A good feasibility study seeks information to cover all aspects of the enterprise including the product, the market, manpower requirements, capital requirements, legal requirements, credit practices in the trade, service of fund, tenders requirement and technical requirements. It is also important when carrying out a feasibility study to find out how profitable the business opportunity is at present.

Most businesses in Nigeria are small and medium scale Enterprises (SMES), they engage less than 100 employees provide goods and services for the citizens and Industries. Small business ought to be the catalyst for Industrialization, poverty reduction and a vital engine of growth in the country.

Feasibility study is an investigation into the potential outcome of the project, but not all entrepreneurs do feasibility studies before going into business. Problems such as difficulty and complication which the process faces in assessing viable and reliable information concerning prospective business are encountered.

This study intends to examine the importance of feasibility studies in enhancing the growth and development of business organization to attain efficiency in business. The study will also try to find out why feasibility studies have showed its relevance and importance in starting up business organization.

\subsection{Objectives Of The Study}

The main objective of this work is to assess the impact of feasibility studies in enhancing the growth and development of business organizations in Nigeria. The specific objectives are to;

(i) Identify the problems of business feasibility studies. 
(ii) Find out the types of information contained in a feasibility study.

(iii) Evaluate the impact of feasibility study information in organizations.

(iv) Assess the market for prospective business plan; Whether the final product will benefit its intended

users

(v) To ascertain if a system development project can be done; is it possible, justified, and whether there is a preferred alternative.

(vi) To compare the performance of businesses which engage in feasibility study and those without it

\section{Review Of The Study}

So many writers have written about feasibility study but the fact still remains that studies or writings on such a subject is a continuous process. Especially as the argument continue whether such a relatively expensive, cumbersome and complex activity is necessary for the success of businesses in a developing world.

Feasibility study is an investigation into the likely potential outcome of a project. It is a planning document which is used to develop an acceptable business plan for the project. As a planning document, it requires a number of decisions for a successful project. After identifying an investment opportunity it is necessary to determine whether the idea is practicable and profitable. An entrepreneur needs to ask practical questions when making feasibility study. Questions like will the idea be practicable? Does a market exist for the product you intend to make? What is the nature of competition in the market? What will be the probable market share? Will the technology (machines) needed be available locally or will it be imported? What about the raw materials? What is the financial requirement of the project? Can I provide all the capital? What market strategy can I adopt? What type of personnel (in terms of skills) will be required? What will be the production process? These questions could go on depending on what the entrepreneur can conceptualize.

Feasibility study report is a technical document therefore; it is complicated and needs the services of a management consultant. A writer of feasibility study has to be vast in different aspects that needs to be treated in the report and also needs to know about the market, marketing and the economic environment as a whole.

\subsection{What Is A Feasility Study}

Feasibility study is an analysis of the viability of an idea; the feasibility study focuses on helping to answer the essential question of "should we proceed with the proposed project idea [1] A feasibility study is also a preliminary study undertaken to determine and document projects viability [2]. The term feasibility study is also used to refer to the resulting document. The result of such a study will be used to make decisions whether to proceed or not with the project. If it indeed leads to the project being approved, it will - before the real work of the proposed project starts- be used to ascertain the likelihood of the project's success. It is also a feasibility analysis, because it analyses possible alternative solutions to a problem and a recommendation of the best alternative is chosen. It can decide on whether an order processing be carried out by a new system more efficiently than the previous one.

A feasibility study is a test against a set of criteria to determine the viability of a proposed venture. These criteria requires a detailed understanding of our product and service, the industry or firm the venture will be competing with, the nature of the market and the amount of capital needed to kick-start the venture.

A basic feature of a feasibility study is that it should provide a thorough analysis of the business opportunity including a look at possible road block that may stand in the way of the co-operative success. The outcome of feasibility will indicate whether or not to proceed with the proposed venture. If the results of the feasibility study are positive, then the cooperative can proceed to develop a business plan. If the result shows that the project is not a sound business idea, then the project should not be pursued.

The main goal of a feasibility study is to assess the economic viability of the proposed business. The feasibility study needs to answer the question "Does the idea make economic sense?[3] identifies main functions of a feasibility report, they include: an aid in business planning which involves an examination of all aspects of the intended business such as cost, sources of suppliers, technical data and expected profit. Secondly, it gives an evaluation of new business ventures for determining feasibility and profitability. Lastly, it provides the basis for the bankers, promoters, suppliers of goods, the various government agencies on which to make their decisions on how to assist the business.

A feasibility study helps an entrepreneur to know whether it is achieving set targets or not and where they are not being met, adjustments should be made [4]. An Entrepreneurship study typically identifies preexisting entrepreneurs and ventures. Few follow the process from idea stage to the ultimate decision to initiate the venture. Research on intentions clearly demonstrates that the intentions are the single best predictor of planned behaviors. Starting a business is hardly an example of simple stimulus-response behaviour. Entrepreneurship is clearly a process where intentionality is central [5]. Relatively, few studies explicitly test theory-driven process models in entrepreneurship or in strategic management. An intentions model offers a coherent and robust framework for pursuing a better understanding of entrepreneurial process. One intention- 
lised model is shapero's model of the entrepreneurial event $(1975 ; 1982)$ which hypothesizes that the intent to start a business derives from perceptions of both feasibility and Desirability and from a propensity to act upon opportunities. Shapero's intention based model of the decision to initiate a new venture (1975) has never been explicitly tested. Imperial support for the model would demonstrate the value of the intentions approach, providing a useful framework for researchers, teachers and practitioners. Thus, it is encouraging to note the increased interest in studying entrepreneurial intentions .According to [6] a business plan as a quintessential part of a feasibility study is a document prepared by the entrepreneur that describes all the relevant external and internal elements involved in starting a new venture. It is often an integration of functional plans such as marketing, finance, manufacturing and human resources. As in the case of [7] it addresses the integration and coordination of effective business objectives and strategies when the venture contains a variety of products and services. It also addresses both short term and long term decision making for the first three years of operation. Thus, the business plan often referred to as the game plan or road map, answers the questions, where am I now? How potential investors and suppliers etc will view the viability of the business. The business plan although often critised for being " dreams of glory", is probably the single most important document to the entrepreneur at the start-up stage. Potential investors are not likely to consider investing in a new venture until the business plan has been completed. In addition, business plan helps maintain the perspective for the entrepreneur of what needs to be accomplished [7]

At this juncture, it is important to state that most businesses in developing economies are small and medium scale enterprises and Nigeria is not an exception [8]. For this reason, many economies have come to realize the value of feasibility studies to the survival of businesses. They are seen to be characterized by dynamism, witty innovations, efficiency and their small size allows for faster decision making. With this fact, a good intentioned feasibility study can result in enhancing the level of the growth and development of the business organization.

It is however tempting to overlook the need for a feasibility study. Often, the steering committee may face resistance from potential members on the need to do a feasibility study; many people will feel that they know the proposed venture as a good idea, so why carry-out a costly study just to prove what they already know? The feasibility study is important because it forces the company to put its ideas on proper reasoning and to assess whether or not those ideas are realistic. It also forces organizations to begin formal evaluation, knowing what steps to take next.

[1] opined that a feasibility study should outline in depth the various alternatives examined, the implications, strength and weakness of each. The project leaders need to study the feasibility study and challenge its underlying assumptions. This is the time for the leaders or entrepreneur to be skeptical.

\subsection{A Business Feasibilty Study}

A Business Feasibility Study can be defined as a controlled process for identifying problems and opportunities, determining objectives, describing situation, defining successful outcomes and assessing the range of costs and benefits associated with several alternatives for solving a problems,. The Business Feasibility Study is used to support the decision making process based on a cost benefits analysis of the actual business or project viability. The feasibility study is conducted during the deliberation phase of the formal Business plan. It is an analytical tool that includes recommendations and limitations, which are utilized to assist the decision making when determining if the Business Concept is viable [9]

\subsubsection{The Importance Of A Business Feasibility Study}

It is estimated that only one in fifty business ideas are actually commercially viable. Therefore a business feasibility study is an effective way to safeguard against wastage of further investment or resources [10]. If a project is seen to be feasible from the result of study, the next logical step is to proceed with the full Business plan. The research and information uncovered in the feasibility study will support the business planning stage and reduce the research time. Hence, the cost of the Business Plan will also reduce. A thorough and viability analysis provides an abundance of information that is also necessary for the Business plan. For example: a good market analysis is necessary in order to determine the business concept's feasibility. This information provides the basis for the market section of the Business Plan[11].

Finally, a feasibility study should contain clear supporting evidence for its recommendations. The strength of the recommendations can be weighed against the study's ability to demonstrate the continuity that exists between the research analysis and the proposed business model. Recommendations will be reliant on a mix of numerical data with qualitative and experienced-based documentation. A business feasibility study is heavily dependent on the market research and analysis. A feasibility study provides the stake holders with varying degrees of evidence that a Business Concept will in fact be viable [11] 


\subsection{Components Of Feasibility Studies}

The components of feasibility studies involves questions such as whether the technology needed for the system exist, how difficult it will be to build and whether the firm has enough experience using that technology[12]. The assessment is based on an outline design of system requirement in terms of input, processes, output, fields, programs and procedures. This can be quantified in terms of volume of data, trends, frequency of updating, in order to estimate if new systems will perform adequately or not. [12]

The various components of feasibility studies are divided into several categories and given different opinions by different authors. Thus;

Schedule Feasibility: Here, those undertaking the feasibility study want to answer questions such as; how long will it take to get the technical expertise? We may have the technology, but that doesn't mean we have the skills required to properly apply that technology hence we may need to hire new people or re-train existing systems staff. Whether hiring or training, it will impact the schedule. An organization must try to assess the schedule risk: Given our technical expertise, are the project deadlines reasonable? If there are specific deadlines, are they mandatory or desirable? If the deadlines are not mandatory, the analyst can propose several alternative schedules. What are the real constraints on project deadlines? If the project overruns, what are the consequences? Deliver a properly functioning information system two months late or deliver an error-prone, useless information system on time? Missed schedules are bad, but inadequate systems are worse!

According to Thompson, Hoagland and Williamson, this involves questions such as how much time is available to build the new system, whether it interferes with normal business operations, type and amount of resources required etc. Contingency and mitigation plans should also be stated here so that if the project does over run, the company is ready for this eventuality. A project will fail if it takes too long to be completed before it is useful. Typically, this means estimating how long the system will take to develop, and if it can be completed in a given period using methods like the payback period.

To O'Brien, the schedule feasibility has to look into operations of the venture in relation to management support, employee, customers, supplier's acceptance and government and other requirements.

Operational Feasibility: How do end-users and managers feel about the problem you identified and the alternative solutions you are exploring? The business must evaluate: Not just whether a system can work but also whether a system will work. Any new solution might meet with resistance: Does management support the project? How do the end users feel about their role in the new system? Which users or managers may resist (or not use) the system? People tend to resist change. Can this problem be overcome? If so, how? How will the working environment of the end users change? Can or will end users and management adapt to the change? These technical questions have to be answered if these businesses have to survive.

To [6], this describes the ventures form of ownership that is proprietorship, partnership or cooperation. If partnership, what are the terms or agreement? If incorporated, who are the principal shareholders and how much stock do they own? It is also helpful to have an organizational chart indicating the line of authority and responsibilities of members of the organization. O'Brien; is of the view that an organizational feasibility should contain how well the proposed system supports the business priorities of the organization.

Market/Financial and economic feasibility: This mostly deals with cost benefit analysis. Economic feasibility answers questions such as: Is the project justified (i.e. will benefits outweigh costs)? Can the project be done within given cost constraints? What is the minimal cost to attain a certain system? Which alternative offers the best return on investment? Selecting among alternative financing arrangements, (rent/lease/purchase). The difficulties of economic feasibility is that benefits and costs can both be intangible, hidden and/or hard to estimate and also ranking multi-criteria alternatives can be cumbersome. A business plan needs to be based on the market forces that could affect the commercial viability of the business. Internal projects must establish the cost-effectiveness of the proposed system that is if the benefits do not outweigh the cost, then it is not worth going ahead. This includes a cost benefit analysis [12]. To O'Brien, the economic feasibility should be cost saving, increase in revenue, increase investment requirements and increase profits.

Hirsch and Peter are of the opinion that the financial feasibility should determine the potential investment commitment needed for the new venture and indicate whether the business plan is economically feasible.

Technical Feasibility: Technical feasibility seeks to evaluate the technical capabilities of the organization: Is the proposed technology or solution practical? Do we currently possess the necessary technology? Do we possess the necessary technical expertise, and is the schedule reasonable? Is relevant technology mature enough to be easily applied to our problem? What kinds of technology will we need? 
Some organizations like to use state-of-the-art technology but most prefer to use mature and proven technology. A mature technology has a larger customer base for obtaining advice concerning problems and improvements. Is the required technology available "in house"? If the technology is available, does it have the capacity to handle the solution? If the technology is not available, can it be acquired?

This show how readily available the technology is upon the understanding of the production process. Questions to be answered to ensure technical feasibility are: is technology (machines) readily available? In the course of breakdown, is it possible to have the technicians to maintain them locally? What about infrastructures needed for the project? If these questions can be answered in the affirmative, then the project is technically feasible [12]

[13] on the other hand states that what necessarily should be involved in technical feasibility includes the hardware and software network capability.

\subsection{Why People Don't Prepare A Feasibility Study}

Project leaders may find themselves under pressure to skip the "feasibility analysis" step and go directly to building a business. Individuals from within and outside of the project may push to skip this step according to[1]; given the following reasons;

1) We know it's feasible. An existing business is already doing it.

2) Why to do another feasibility study when one was done just a few years ago?

3) Feasibility studies are just a way for consultant to make money.

4) The feasibility analysis has already been done by the business that is going to sell us the equipment. The reasons given above should not dissuade one from conducting a meaningful and accurate feasibility study. Once decisions have been made, about proceeding with a proposed business, they are often very difficult to change. You may need to live with these decisions for a long time.

Other suggestions why people don't conduct feasibility study Include:

1) Feasibility studies are just a waste of time and other resources.

2) Why not just hire a general manager who can do the study?

\subsection{Who Should Write The Feasibility Study (Plan)?}

A feasibility studies is a plan, a written document prepared by the entrepreneur [13]. It describes all the relevant external and internal elements involved in starting a venture. A feasibility study is prepared by the entrepreneur consulting many other sources in its preparations, these sources could include lawyers, accountants, marketing consultants and engineers whom could be useful in the preparation of different levels or step in the plan.

According to [14], in many instances entrepreneurs will actually hire or offer equity (partnership) to another person who might provide the appropriate expertise in preparing the business plan as well as become an important member of the management team to help determine whether to hire a consultant or to make use of other resources, the entrepreneur can make an objective assessment of his or her own skills.

\subsection{Who Reads The Feasibility Study (Plan)?}

[13] is of the view that a feasibility study plan may be read by employees, investors, bankers, venture capitalist, suppliers, costumers, advisors and consultants. According to [14], whoever reads feasibility study plan should affect its actual content and focus. Different people read a feasibility study for different purposes, the entrepreneur must therefore be prepared to address all their issues and concerns. In one way or the other, the plan must try to satisfy the needs of everyone. However [13], states three perspectives that should be considered in this preparation;

First, the perspective of the entrepreneur who understands better than analyze the creativity and technology involved in the new venture. The entrepreneur must be able to clearly articulate what the venture is all about.

Secondly, if the marking perspective too often, an entrepreneur will consider only the product or technology and not whether someone would buy the product on offer. Entrepreneurs must try to view their business through the eyes of their customers.

Lastly, the entrepreneur should try to view his or her business through the eyes of the investors. Sound projectionists are required. If the entrepreneur does not have the skills to prepare this information, then outside sources can be of assistance.

\section{Methodology}

This section presents the tools, methods and the procedures employed in the collection of data for the purpose of this study. The main issues will include method of data collection and method of data analysis 
showing the relevance of each in carrying out this study so as to result in the stated objectives and significance of the study.

\subsection{Methodology of the Study}

This is a fact finding study and it seeks to find out the impact of feasibility study in enhancing the growth and development of business organizations in Nigeria. It seeks to verify if Nigeria as a developing country needs to engage in feasibility studies in setting up industries as a pre-requisite for the long term survival of such ventures.

For the purpose of this work, the sample size will be taken from a selected number of business entrepreneurs and few individuals who intend to go into business and from the layman's points of view.

A sample size of 60 respondents will be interviewed and administered questionnaires. Information gathered from the respondents would then be analyzed to prove the acceptability of the different hypotheses formulated.

There are two sources of data collection; the secondary and primary sources of data collection. Primary sources of data collection include the administration of questionnaires, personal interviews and observations.

Secondary sources of data collection include the use of publications, journals, internet, magazines and any relevant write-up. A vital source of secondary data is the internet. In the process of carrying-out this study, the use of primary and secondary sources of data was necessary.

Having generated the needed data, the study will proceed to analyze the data collected. In analyzing the data, the statistical tool that will be used to justify the hypotheses is ANOVA. The underlying assumption for the use of this statistical tool is that there must be independent random sampling from the population and that population under study must be normally distributed. The population used for this research satisfies this assumption.

\section{Analysis And Discussion}

This section presents the data collected, its analysis and states the outcome of the analysis based on findings. The purpose of this chapter therefore is to present and process data and obtain information based on analysis so as to achieve the objective of this study.

The initial section consists of simple percentage tables upon which basic and direct suggestions were made. The Justification of the hypotheses was also carried out in this chapter.

\subsection{Discussion}

Based on the data being collected and analyzed in the course of carrying out this study, the research arrived at these findings:

From the study conducted, it was found that most business entrepreneurs are retailers and are into small-scale entrepreneurship. Most of the business entrepreneurs that were administered questionnaires responded positively on whether they had feasibility study before starting up their business or not. However, the study reveals that the entrepreneurs don't engage the services of professionals like consultants, accountants and lawyers in drawing up the business plan. Most of the respondent believes that their business needs a feasibility study as portrayed by most of the respondents have benefitted from one form of feasibility study or the other. Lack of business planning (that is feasibility study), has affected adversely the growth of most businesses.

It also reveals that majority of businesses are not registered with the Corporate Affairs Commission (CAC). The result concluded that business that undertook feasibility studies record significant success than those that did not.

\section{Summary}

In carrying out this study steps were being followed to arrive at the finding on whether feasibility study has an impact on enhancing growth and development of business organizations in Nigeria. The study was subdivided; consisting of introduction, literature review, methodology, data presentation and finally summary of the study. The first chapter consists of introduction of the topic, statement of problems, objectives and significance of the study.

The second chapter looked at various literatures that relate to feasibility study. Here the researcher dealt with theoretical frame work of the practicability of feasibility studies in small and medium scale industries. Most businesses do feasibility studies at their level.

In the third section, method of data collection, sources of data collected and analysis used while chapter four deals with presentation and analysis of data. The last section includes summary of the study, limitation of the study, conclusions and recommendations based on the findings.

\subsection{Conclusions}


Based on data being presented and analyzed in the process of this study, it can be concluded that feasibility study has an impact in enhancing the growth and development of business organizations in Nigeria because a greater fraction of responses from respondents were positive on the impact of feasibility study at every stage of their business.

\subsection{Recommendations}

In referring to the findings of this study, recommendations can be made based on the following suggestions;

- It is important that the services of professionals should be engaged to draw up a comprehensive or detailed plan for the entrepreneur since they have a vast knowledge in this field rather than the entrepreneurs doing it themselves because from the study conducted, it is revealed that entrepreneurs do the feasibility studies themselves. And as a result of this, they fail to establish their businesses in relation to the business plan because of improper preparation of the plan.

- Prospective entrepreneurs should be encouraged by professionals on having a feasibility study because from the study conducted it was found that lack of feasibility study has affected the growth of $51.8 \%$ businesses.

- There is a need for businesses to be registered with the Corporate Affairs Commission (CAC) as the importance of the commission cannot be over emphasized. Government should make it mandatory for every business, no matter how small to register with the CAC. This organization in partnership with other private agents will monitor the growth as well as demise of these businesses. Government can through the agency see how it can assist weak and dying businesses because their information is readily available so that small scale businesses can be guided to grow.

- Infrastructural development should be enhanced by Government, especially power as most businesses affirm that a huge chunk of their budget is eaten up through sourcing energy to run their businesses. There are no easy ways in their feasibility study plan to cut down the soaring cost of energy.

- The private sector should be encouraged by government to engage in continuous and consistent workshops in major Nigerian cities on the need to engage in feasibility studies before and during their business to enhance expansions. Papers presented in such seminars should be made available at all level through government machineries in form of monthly bulletins.

[1]. Hofstrand and Holz-Clause (2008) mclause@ istate.edu

[2]. Cashman and Rosenblatt (2006) System Analysis and Design. www.wikipedia.org

[3]. Oshagbemi, (1983) Feasibility study Report,Ibadan Nigeria

[4]. Nigeriabusinessinfo.com (Feb, 2004) Prospects of Nigerian Small and Medium Scale Enterprises under the Small and Medium Industries Investment Scheme (SMIES).

[5]. Katz J. A\& D.G Armhein (1998) cash flow as a factor in the mortality risk of a Business Paper Presented at the International Council of Small Business, Singapore

[6]. Hisrich peter \& Venkatraman (2002)

[7]. Nigeriabusinessinfo.com (Feb, 2004) Small and Medium Scale Enterprises and Funding in Nigeria.

[8]. Drucker P. F. (1985). Innovations \& Entrepreneurships. New York, Harper Trade.

[9]. L. Gofton \& M. Ness (1997) business market research, kogan page London pp 165

[10]. Hoagland Horbes. and Williamson Landslay (2000) Feasibility Studies, Kentucky, University of Kentucky.

[11]. Hoagland and Williamson (2000) http://www.uky.edu/agecon/pub/ext-other/feasibilitystudy.pdf

[12]. Thompson Andrew (2003) Overview of a Business plans. Perth, Best Entrepreneur.

[13]. Wickham Perth (2004); Strategic Entrepreneurship, Essex, Pearson Education.

[14]. Kruger, Norris (2008) The Impact of Prior Entrepreneurial Exposure on Perceptions of New venture Feasibility and Desirability. Journal Article Excerpt.www.wikipedia.org 